OPEN ACCESS

Edited by:

Bruno Sainz, Jr.,

Autonomous University of Madrid,

Spain

Reviewed by:

Xin Tian,

Shaanxi Academy of Traditional

Chinese Medicine, China

Feng Wei,

Tianjin Medical University Cancer

Institute and Hospital, China

*Correspondence:

Ramin Radpour

ramin.radpour@dbmr.unibe.ch

Specialty section:

This article was submitted to

Gastrointestinal Cancers,

a section of the journal

Frontiers in Oncology

Received: 19 June 2020 Accepted: 08 October 2020 Published: 09 November 2020

Citation:

Forster S and Radpour R (2020) Molecular Immunotherapy:

Promising Approach to Treat

Metastatic Colorectal Cancer by

Targeting Resistant Cancer

Cells or Cancer Stem Cells.

Front. Oncol. 10:569017.

doi: 10.3389/fonc.2020.569017

\section{Molecular Immunotherapy: Promising Approach to Treat Metastatic Colorectal Cancer by Targeting Resistant Cancer Cells or Cancer Stem Cells}

\author{
Stefan Forster ${ }^{1,2}$ and Ramin Radpour ${ }^{1,2 *}$ \\ 1 Tumor Immunology, Department for BioMedical Research (DBMR), University of Bern, Bern, Switzerland, ${ }^{2}$ Department of \\ Medical Oncology, Inselspital, Bern University Hospital, University of Bern, Bern, Switzerland
}

The immune system is able to recognize and eliminate tumor cells. Some tumors, including colorectal cancer (CRC), induce immune tolerance via different mechanisms of "immunoediting" and "immune evasion" and can thus escape immune surveillance. The impact of immunotherapy on cancer has been investigated for many years, but so far, the application was limited to few cancer types. Immuno-oncological therapeutic strategies against metastatic colorectal cancer ( $\mathrm{mCRC})$, the adaptive immune system activating approaches, offer a high potential for adaptation to the great heterogeneity of CRC. Moreover, novel treatment approaches are currently being tested that might specifically target the disease initiating and maintaining population of colorectal cancer stem cells (CSCs). In this review, we aim to summarize the current state of immune-oncology and tumor immunotherapy of patients with $\mathrm{mCRC}$ and discuss different therapeutic modalities that focus on the activation of tumor-specific T-cells and their perspectives such as tumor vaccination, checkpoint inhibition, and adoptive T-cell transfer or on the eradication of colorectal CSCs.

Keywords: colorectal cancer, cancer stem cells, metastasis, cancer therapy, immunotherapy, immunecheckpoint inhibitors

\section{INTRODUCTION}

Colorectal cancer (CRC) is the third most common cancer and the fourth most common cause of cancer-related deaths (1). Worldwide, 1.4 million people fall ill every year and almost 700,000 people die due to metastatic CRC (mCRC) $(2,3)$. The majority of CRC patients develop metastases during the course of the disease, which is associated with a dismal prognosis and a 5 -year survival rate of less than $10 \%$ (4). Approximately $15-25 \%$ of patients present with liver metastases at the time of initial diagnosis and 30\% develop liver metastases later in the disease period (5). Despite different systemic therapy advances, more than $80 \%$ of patients with mCRC die within 5 years upon diagnosis. Currently, the majority of $\mathrm{mCRC}$ patients are treated with a combination of a biological agent together with a cytotoxic drug. While chemotherapy combined with surgical rehabilitation 
and/or radiological interventional procedures are the treatment of choice, several clinical parameters (e.g., age, comorbid illness, tumor localization, tumor burden, and resectability) influence the treatment options. Among those clinicopathological parameters, molecular characteristics of CRC including B-raf proto-oncogene, serine/threonine kinase $(B R A F)$, human epidermal growth factor receptor 2 (HER2), microsatellite instability (MSI), and rat sarcoma homolog family (RAS) are important therapy determinants $(5,6)$. Resection and (neo) adjuvant chemotherapy (CTx) can improve 5-year survival rates (7). However, the recurrence rate is $40-75 \%$, of which $50 \%$ affect the liver (8). With extensive liver metastases, surgical procedures using established technologies are often no longer possible, and the option of surgical rehabilitation is reserved for only a minority of these patients (9). The presence of nonresectable colorectal metastases implies a significantly worse prognosis. Under this condition, the palliative CTx remains the only therapeutic option left (10). In order to prevent tumor progression, immunotherapy approaches are proposed. In principle, the procedure should be determined for all patients with mCRC in interdisciplinary tumor boards.

Cancer stem cells (CSCs) represent a minor fraction of the bulk tumor cell population that could potentially reconstitute and propagate the disease. CSCs are found in different tumor types including colorectal tumors (11-14). CSCs can also induce tumors in foreign tissues (xenograft models); they diverge in different tumor types by their specific cell surface markers and have the potential to rebuild heterogeneous tumor tissue. In addition, CSCs possess stem cell properties such as self-renewal and quiescence that are regulated by cell-intrinsic and cellextrinsic mechanisms (15). CSCs are mainly resistant to conventional therapies such as chemotherapy, irradiation and against immune attack; therefore, they are the main initiator of cancer relapse after primary treatment. This may be due to the different escape mechanisms of CSCs and/or due to the protective mechanisms of the microenvironment, e.g., the tumor niche $(14,16)$. For this reason, targeting and eradication of CSCs have been some of the main challenges in cancer treatment (17).

The immunotherapy of tumors has established itself as an important pillar of oncological treatment. Distinction of immunebased therapies is made according to their mechanism of action between active and passive immunotherapies. Decades of preclinical and clinical research suggest that the immune system is able to prevent tumorigenesis and fight cancer (18). The immunosuppressive function of inhibitory cytokines/chemokines as well as the complex microenvironment of CRC, reduce the immune function to promote CRC growth (6).

In CRC, an accumulation of driver mutations leads to the formation of the tumor-initiating cells, that represent a "foreign tissue" to the immune system. To date, the immunotherapy benefit is mainly confined to a small subset of patients with hyper mutated microsatellite instability-high (MSI-H) tumors such as CRC patients with deficient mismatch repair (dMMR) who only represent a small proportion of $\mathrm{CRC} / \mathrm{mCRC}$ patients. Those dMMR tumors carry a high level of somatic mutations and therefore are considered as being highly immunogenic (6).

\section{ACTIVE AND PASSIVE IMMUNOTHERAPIES}

In general, immunotherapies can be divided into active and passive forms. The difference is whether the molecule used for treatment works by activating the immune system or is only part of the immune system itself (Figure 1).

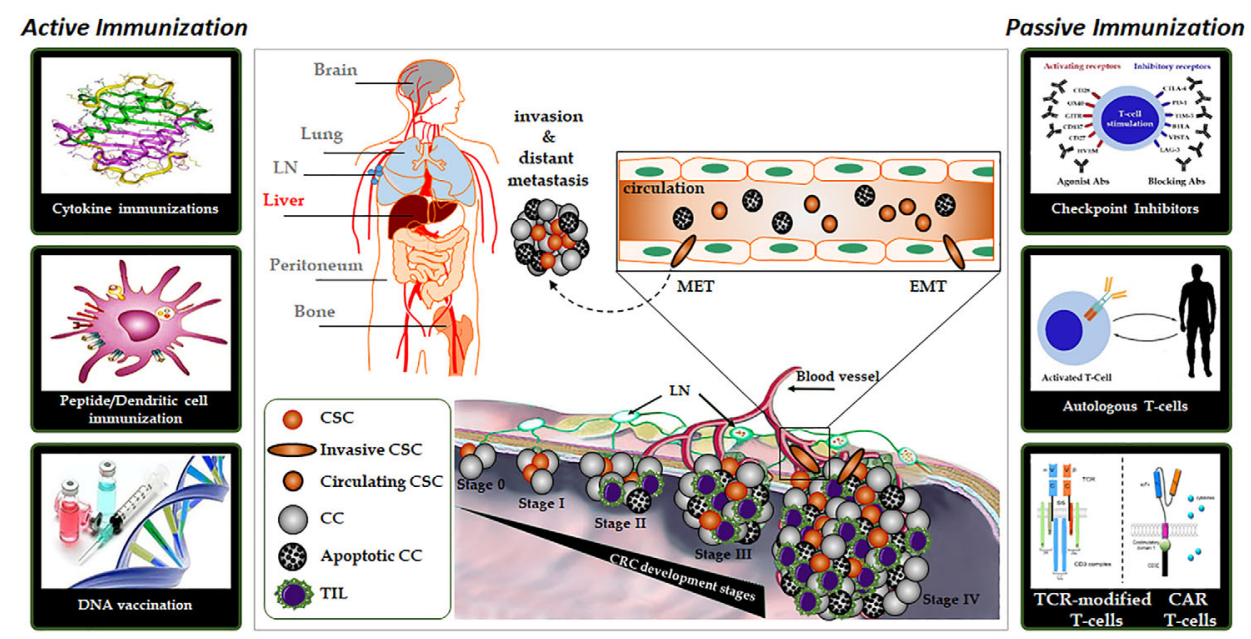

FIGURE 1 | The complex organization of cancer initiation, progress, and distant metastasis for colorectal cancer and important active or passive immunotherapy approaches. Abbreviations: CC, Cancer cell; CSC, Cancer stem cell; EMT, Epithelial-mesenchymal transition; MET, Mesenchymal-epithelial transition; LN, Lymph nodes; TIL, tumor-infiltrating lymphocytes. 


\section{ACTIVE IMMUNOTHERAPIES}

\section{Cytokines}

The discovery, cloning and recombinant production of intercellular messengers, known as cytokines, was initially marked by the hope that they could be used to treat tumors (19). However, it became clear that sometimes their high potency could cause systemic side effects before inducing a tumordirected effect. For example, interleukin-1 $\beta$ (IL1B) already leads to fever attacks at the nanogram level (20). Only three representatives of this category are currently clinically used in oncology: interleukin-2 (IL2), interferon- $\alpha$ (IFNA1), and tumor necrosis factor- $\alpha$ (TNF). IL2 is predominantly involved in the activation of various lymphocyte populations [NK (natural killer) and T-cells]; it induces and reactivates antitumoral immune responses. Although this type of therapy shows numerous side-effects, treatment with IL2 resulted in persistent remissions in a subset of patients with mCRC (21). Moreover, IL2 is frequently used in immunotherapy combinations involving autologous T-cells to induce T-cell expansion (22). IFNA1 is approved under defined conditions for the treatment of a variety of tumor types including $\mathrm{mCRC}$ alone or in combinations (23). The recombinant IFNA1 mimics a viral infection and leads to an antiviral program in the patient and its tumor tissue. In this case, IFNA1 presumably acts directly on the tumor cell and on its environment and thereby unfolds its therapeutic effect (24). The therapeutic window is narrow due to the high toxicity of the substance. TNF triggers both an antitumor inflammation and a direct cytotoxic effect. However, it has been reported that Th17-type cytokines (including TNF and IL6) promote CRC growth via activation of NFKB1 and STAT3 genes (25).

\section{Tumor Vaccination}

Vaccination leads to the detection of tumor antigens by the immune system, subsequently triggering a specific antitumor immune response. In tumor vaccination, the presentation of tumor antigens allows effective activation of tumor-specific T-cells (i.e., $\mathrm{CD}^{+}$cytotoxic T-cells), thereby inducing or increasing an antitumor immune response.

\section{Agonists for Pattern Recognition Receptors}

Pattern recognition receptors are important components of the innate immune response. They are used for the rapid detection of bacteria and viruses via the binding to specific patterns of these pathogens. This triggers pro-inflammatory signaling cascades that first mobilize soluble and cellular components of the innate immune response. The activation of pattern recognition receptors may also lead to the induction of an adaptive, acquired immune response. With the discovery of these receptors and their ligands, it was suggested that such agonists could be used for tumor therapy. As an example, catumaxomab binds on the one hand to the T-cell antigen $\mathrm{CD} 3$ and on the other hand to EPCAM ("epithelial cell adhesion molecule"), a tumorassociated antigen (26). Via its CD3 binding arm, catumaxomab activates T-cells by cross-linking them with tumor cells thus leading to tumor cell lysis. In addition, catumaxomab has also a functional $\mathrm{Fc}$ domain. Via this $\mathrm{Fc}$ domain, catumaxomab binds to antigen-presenting cells, possibly promoting the development of an immunological memory. The second approved product is blinatumomab, a bispecific antibody that binds to $\mathrm{CD} 3$ and CD19. This has the peculiarity that it consists of two so-called "single chain domains" (27). Catumaxomab and blinatumomab are examples of how T-cells can be targeted against tumors.

\section{Target Antigens for Tumor Vaccination}

In tumor vaccination, highly complex, polyvalent and inaccurately characterized antigenic mixtures or well-defined antigens $(\mathrm{Ag})$ can be used alone or in combination as vaccines. Frequently used Ags in clinical studies are Ag overexpressed in tumor cells, so-called tumor-associated antigens (TAA), cancertestis Ag and oncofetal Ag (Table 1). Although tumor-individual and patient-specific Ags, so-called neoantigens, have been known for a long time, they can only be exploited by highthroughput screening/sequencing methods including the help of dedicated software and bioinformatic algorithms to predict the peptide binding avidity to MHC molecules (28). Vaccination strategies against patient-specific neoantigens appear promising today. The concept of neoantigen vaccines is currently being investigated in different clinical studies for CRC (Table 2).

TABLE 1 | Potential tumor antigens for CRC vaccination.

\begin{tabular}{|c|c|c|c|}
\hline Antigen & Examples & Strength & Weakness \\
\hline $\begin{array}{l}\text { Tumor-associated } \\
\text { antigens (TAA) }\end{array}$ & $\begin{array}{l}\text { PSG2 (CEA), } \\
\text { ERBB2 (Her2/neu), } \\
\text { GP100, MLANA (MART-1), } \\
\text { MUC1, PSA, Tyrosinase }\end{array}$ & $\begin{array}{l}\text { Immunogenic with strong expression in many } \\
\text { tumors and low expression in normal tissue }\end{array}$ & $\begin{array}{l}\text { It is possible that only } \mathrm{T} \text {-cells with weak avidity are } \\
\text { activated }\end{array}$ \\
\hline $\begin{array}{l}\text { Mutated tumor-specific } \\
\text { antigens (neoantigens) }\end{array}$ & $\begin{array}{l}\text { TP53, RAS, patient-specific } \\
\text { mutations }\end{array}$ & $\begin{array}{l}\text { Activation of T-cells with high avidity and } \\
\text { effectiveness }\end{array}$ & $\begin{array}{l}\text { Identification so far very cost-intensive, therefore not } \\
\text { yet applicable for the routine application }\end{array}$ \\
\hline Cancer-testis antigens & $\begin{array}{l}\text { MAGE (e.g., MAGEA3), } \\
\text { CTAG1B }\end{array}$ & Immunogenic with expression in numerous tumors & $\begin{array}{l}\text { It is possible that only T-cells with weak avidity are } \\
\text { activated }\end{array}$ \\
\hline Oncofetal antigens & AFP & $\begin{array}{l}\text { Immunogenic with strong expression in many } \\
\text { tumors, no expression in adult normal tissue }\end{array}$ & $\begin{array}{l}\text { It is possible that only } \mathrm{T} \text {-cells with weak avidity are } \\
\text { activated }\end{array}$ \\
\hline
\end{tabular}

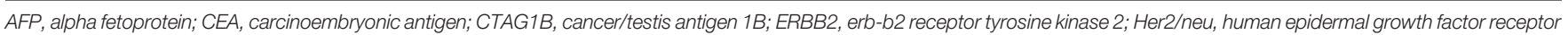
2; MART-1, melanoma antigen recognized by T-cells; GP100, glycoprotein 100; MAGE, melanoma antigen-encoding gene; MUC1, mucin 1; PSA, prostate-specific antigen; PSG2, pregnancy-specific beta-1-glycoprotein 2; TP53, tumor protein P53. 
TABLE 2 | Overview of immunotherapy approaches and clinical trials in CRC/mCRC.

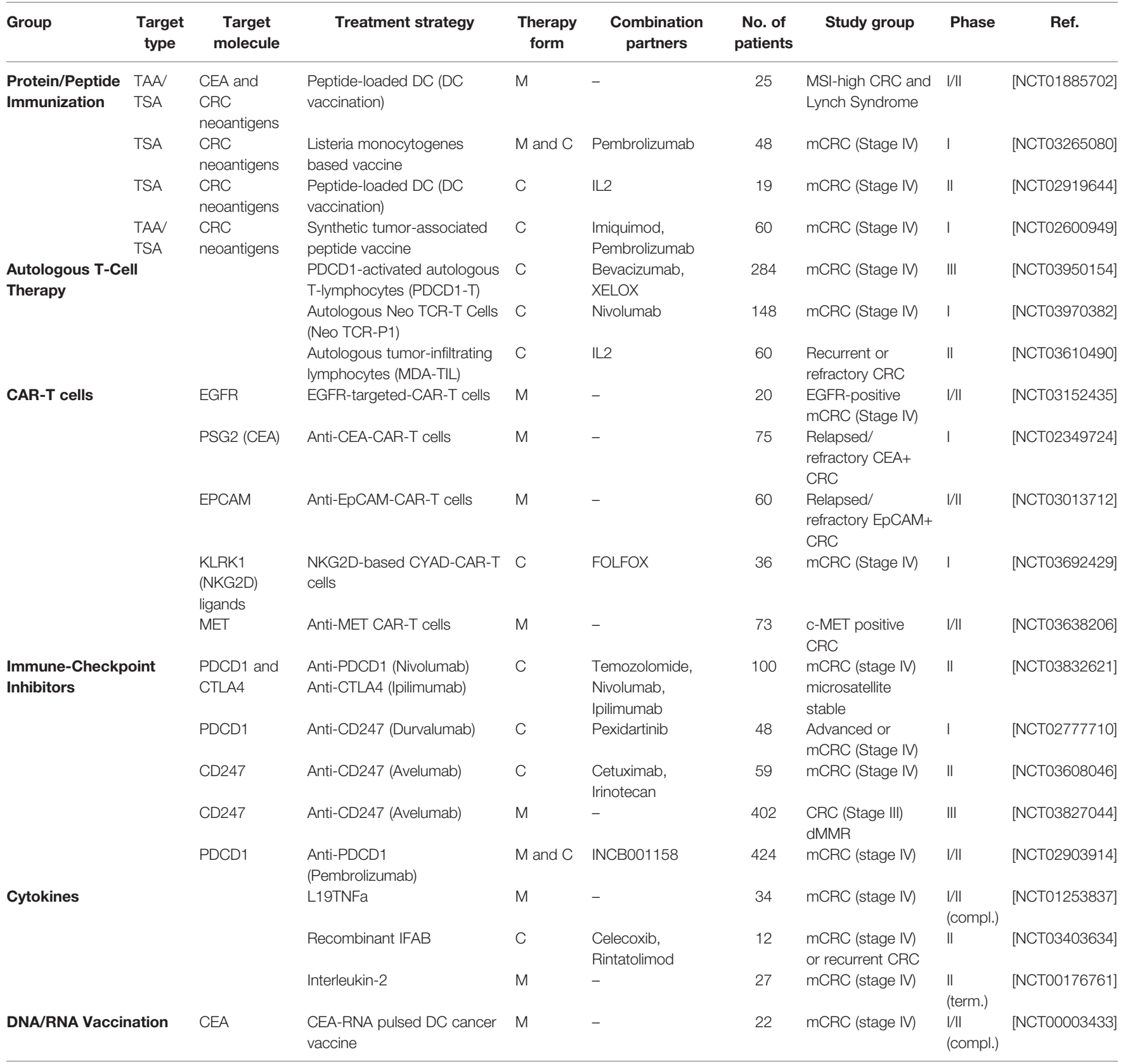

C, combination therapy; CAR-T cells, chimeric antigen receptor T cells; CD247 (PDL-1), programmed cell death-ligand1; Compl., completed; CRC, colorectal cancer; DC, dendritic cells; IMMR, deficient mismatch repair; IFAB, interferon Alfa-2b; IL2, interleukin-2; KLRK1, killer cell lectin-like receptor K1; M, monotherapy; mCRC, metastatic colorectal cancer; MSI, microsatellite instability; PDCD1, programmed cell death-rpotein-1; PSG2, pregnancy-specific beta-1-glycoprotein 2; TAA/TSA, tumor-associated antigens/tumor-specific antigens; TIL, tumor-infiltrating lymphocytes; term, terminated.

\section{Tumor Vaccination Strategies}

Tumor vaccination involves a wide range of approaches, which can be essentially divided into three strategies: peptide/protein vaccines, cell-based vaccines, and genetic vaccines. In peptide/ protein vaccination, peptides or proteins from tumor antigens are administered as vaccines in combination with different adjuvants (29). Previous peptide/protein vaccination studies have often used MHC class I-restricted peptide epitopes from TAA. However, most peptide/protein-based vaccine research approaches have yielded disappointing results so far and have not been further developed until their clinical testing (30).

In cell-based vaccination, cells or cell lysates serve as a vaccine. Here, vaccination strategies are mainly based on autologous dendritic cells (DC), the most effective antigen presenting cells (APC). In this strategy, DC progenitor cells are taken from the blood of the patient, are cultivated in vitro and stimulated by the addition of tumor-specific antigens. These pre-treated cells are then reinfused into the patient (30). Several 
DC/APC-based vaccination strategies are in advanced clinical trials. Other cell-based vaccine approaches, such as vaccination with autologous or allogeneic ex vivo irradiated tumor cells, have shown disappointing results in previous studies (30).

Genetic vaccination approaches (DNA/RNA/virus-based) induce somatic cell or DC expression of tumor antigens and their presentation in the context of MHC class I and II molecules. This can trigger a direct immune response against tumor cells (30). Initial clinical trials of RNA-based vaccine approaches are promising and suggest a superior side-effect profile over the other genetic vaccines (DNA/virus-based vaccines) (Figure 2,

Table 2).

Over many years, the potent stimulatory effects of Toll-like receptors (TLRs) on the immune system have urged efforts aiming to develop immune vaccines that use TLR agonists as immunological adjuvants $(31,32)$. Motolimod (VTX-2337) and resiquimod (R848) are TLR-8 and TLR-7/TLR-8 agonists respectively, that deliver adjuvant-like signals to APCs. Both are derivatives of first generation immunomodulatory agents like imiquimod, which was originally approved by the US Food and Drug Administration (FDA) to treat genital warts and actinic keratosis. VTX-2337 and R848 are currently being investigated as potential immune system stimulators for the treatment of various tumor types (including CRC and mCRC). They might be particularly considered effective in combination therapies together with cancer cell lysate-based, dendritic cell-based, DNA molecules-based or peptide-based vaccines (31). The CD200 receptor (CD200R) inhibits immune activation upon binding to its ligand CD200 that is often expressed on tumor cells to diminish anti-cancer immune response $(33,34)$. Previous studies have shown that the intratumoral administration of R848 inhibits tumor growth and decreases CD200R expression on tumor-infiltrating immune cells in a syngeneic CT26 colon carcinoma mouse model. These results indicate that the antitumor activity of the TLR-7/TLR-8 agonist (R848) is mainly driven by an anti-CD200R effect causing changes in the tumor microenvironment (TME) (32).

DNA motifs containing unmethylated cytosine-guanosine oligodeoxynucleotides (CpG-ODN) have an immunostimulatory function and can induce antitumor immune responses mediated by the innate and the adaptive immune system through TLR-9 signaling upon activation of APCs. CpG-ODNs promote the maturation of APCs and support the generation of antigenspecific B cells and cytotoxic T lymphocytes $(35,36)$. In humans, TLR-9 is mainly expressed by B cells and plasmacytoid dendritic cells. Several experimental models have shown that immune modulation by a TLR-9 agonist (e.g., CpG-28 or MGN1703) can activate both innate and adaptive immunity resulting in a significant tumor rejection; particularly when injected directly into the tumor (37, 38). Additionally, intratumoral injection of CpG-ODN enhances the host's response against cancer cells by reducing the immunosuppressive activity of myeloid-derived suppressor cells (MDSCs) (39). CpG-ODN treatment can also increase the TNF production in DCs or peripheral blood mononuclear cells (PBMCs) (40).

Multiple clinical studies have been initiated and first analyses suggest a certain efficacy of vaccination-based approaches in different tumor types. However, the overall clinical success has been low especially for CRC (41). To date, there are no clinically approved vaccination therapies in CRC or mCRC treatment, and those that are tested in different clinical trials can induce a therapeutic response only in a minority of patients (about 510\%) (30).

The reasons why previous vaccination strategies did not produce satisfactory clinical response rates are mainly explainable by the decreased reactivity of the immune system



FIGURE 2 | Illustration of adoptive T-cell transfer. Adoptive transfer of TIL (right). Adoptive transfer of TCR and CAR-modified T-cells (left). CAR, chimeric antigen receptor; CC, cancer cell; CSC, cancer stem cell; TCR, T-cell receptor; TIL, tumor-infiltrating lymphocytes. 
to tumor-associated self-antigens due to tolerance and immunosuppressive mechanisms (30). In addition, previous research studies have used single Ag or combinations of only a few Ags as the basis for tumor vaccination strategies. However, according to most recent studies and guidelines multi-epitope vaccines are considered superior to single Ag based vaccines (41). There is also emerging evidence that tumor vaccination appears to be more effective when the tumor burden is still at a low level. Previous studies, however, were mostly performed on advanced cancer stages or metastatic forms (41).

\section{PASSIVE IMMUNOTHERAPY}

\section{Antibodies}

Monoclonal antibodies are the longest used form of immunotherapy. They are part of the treatment regimens for many tumors including mCRC. Antibodies, directed against the tumor or tumor-associated structures could mediate their effect via induction of cell death, activation of the complement system, activation of effector cells of the immune system via the Fc part of the antibody or opsonization ("labeling") of tumor cells, and facilitation of phagocytosis by myeloid cells (42).

\section{Immune-Checkpoint Inhibitors}

Checkpoint inhibitors (CHI) have revolutionized tumor therapies in recent years. Their discovery is considered as one of the most important immunotherapy innovations of the last decade. Several compounds targeting these molecules are already in development (43).

The immune system has numerous co-stimulatory and inhibitory signaling pathways that help to regulate the strength of an immune response and prevent autoimmune reactions. The inhibitory signaling pathways, so-called immune-checkpoints, cause downregulation of T-cell activation or effector function and play a central role to protect our body from excess immune and T-cell responses. Tumor cells use upregulation of immunecheckpoints to escape immune system recognition (immune evasion) and protect themselves from T-cells and the immune system. This knowledge led to the development of CHIs. These inhibitors are monoclonal antibodies (mAbs) directed against immune-checkpoint receptors or ligands; thereby, resolving the physiological "immune brakes." Because CHI modulate the immune response, they clinically show a different type of response than conventional oncology therapeutics. CHI may initially lead to an apparent phase of tumor growth that is followed by tumor regression (44). The described side-effects of CHI can also largely be explained by the immune-stimulatory mode of action, which can cause a misguided immune response. However, $\mathrm{CHI}$-side effects can be treated well (44).

Three representatives of checkpoint molecules play an essential role in oncology: PDCD1 ["programmed cell death protein 1 (PD1)"], CD247 ["programmed cell death-ligand 1 (PD-L1)”], and CTLA4 ("cytotoxic T-lymphocyte antigen-4"). All three molecules were tested and validated as targets for blocking antibodies. CTLA4 belongs to the first generation of CHI. CTLA4 is expressed on activated cytotoxic T-cells and acts as the antagonist of the costimulatory receptor CD28 that is required in T-cell activation. The blockade of CTLA4 thus leads to a de novo generation and expansion of T-cells. The mAb, ipilimumab, binds to CTLA4 and subsequently activates antitumoral effects in the early phase of T-cell activation within the lymph nodes. Another CTLA4 inhibitor, tremelimumab, is currently in clinical development (45).

The target genes of the second generation of $\mathrm{CHI}$ are the checkpoint receptor PDCD1 and its ligands CD247 (B7-H1) and PDCD1LG2 (B7-DC or PD-L2). PDCD1 plays a key role in the regulation and maintenance of the balance between $\mathrm{T}$-cell activation and immune tolerance (45). The PDCD1/CD247 axis plays a pivotal role in the effector function of T-cells, i.e., in T-cells residing within (tumor) tissue. It protects cells from excessive T-cell activation by the expression of CD247 on the cell surface that interacts with PDCD1 expressed by T-cells. Thus, the blockade of the PDCD1/CD247 axis would reactivate an existing T-cell response. Several $\mathrm{mAbs}$ against PDCD1 (pembrolizumab, lambrolizumab, nivolumab, and pidilizumab) and its major ligand CD247 (BMS-936559, MPDL3280A, etc.) are currently in clinical development to target a variety of tumors including mCRC. The most advanced substances are pembrolizumab and nivolumab. Numerous other checkpoint molecules are the subject of intensive preclinical research: Hepatitis A virus cellular receptor 2 [HAVCR2; known as Tcell membrane protein 3 (TIM-3)], lymphocyte activation gene 3 (LAG3) (Table 2).

Little oxygen is beneficial for tumors by preventing tumor cells from T-cell interaction. Moreover, hypoxia counteracts the desired effects of CHI, such as PDCD1 or CTLA4 inhibitors leading to $\mathrm{CHI}$ resistance of tumor cells (46). In this regard, the substance evofosfamide ( $\mathrm{TH}-302)$, an alkylating prodrug that is activated by a lack of oxygen supply, is currently being clinically tested (47).

It is already evident that some types of tumors such as melanoma, lung, kidney, or bladder carcinoma and Hodgkin's lymphoma respond better to immunotherapy with $\mathrm{CHI}$ than other types of tumors (e.g., tumors of the gastrointestinal tract and pancreatic carcinoma) (30). The two PDCD1 inhibitors (pembrolizumab and nivolumab) have been evaluated alone or in combination with a CTLA4 inhibitor in patients with chemorefractory mCRC in the frame of several clinical studies (Table 2). As results, patients had an improved therapy response rate and around $60-70 \%$ disease control $(48,49)$. Interestingly, the response to $\mathrm{CHI}$ was irrespective of CD247 expression within tumor cells. Further, the response rate was independent from the history of Lynch syndrome and BRAF or KRAS mutation status (50). Although CHI (including PDCD1 blockade or antiCTLA4) in patients with dMMR/MSI-H mCRC significantly increase the antitumor activity of tumor specific $\mathrm{CD}^{+} \mathrm{T}$-cells with highly durable tumor response, they are associated with virtually no activity in patients with $\mathrm{pMMR} /$ non-MSI-H mCRC $(51,52)$.

Tumor-infiltrating $\mathrm{CD}^{+} \mathrm{Foxp}^{+}$regulatory $\mathrm{T}$ (Treg) cells are known as potent immunosuppressive cells. Treg cells represent 
one of multiple TME components that help cancer cells to evade the immune system $(53,54)$. Accumulation of Tregs within tumor tissues and the subsequent high ratio of Tregs to effector T (Teff) cells, is correlated with poor prognosis of cancer patients suffering from different types of malignancies, including CRC (55). Thus, several cancer immunotherapy approaches purging the activity of $\mathrm{CD} 4^{+}$Foxp $3^{+}$Treg cells by either depletion of or down-regulating their immunosuppressive function using immune-checkpoint inhibitors such as antiCTLA-4 monoclonal antibody therapy. This approach has become an effective cancer immunotherapy attributing to depletion of Tregs in tumors (54).

\section{COMBINATION THERAPY}

Despite good clinical results, many patients do not respond to single $\mathrm{CHI}$ treatment. However, it might be possible to optimize overall survival rates through appropriate therapy combinations. The spectrum of combination partners ranges from further $\mathrm{CHI}$ and vaccines, via radiotherapy and chemotherapies, to targeted therapeutic approaches (56). The combination of ipilimumab (CTLA4 inhibitor) and nivolumab (PDCD1 inhibitor) results in a synergistic effect improving progression-free survival compared to monotherapy with nivolumab or ipilimumab in tumors with mismatch-repair deficiency (CRC and mCRC) $(48,51)$. A cohort of 119 patients showed disease control rates of around $80 \%$ and overall response rates of more than $50 \%$ upon combination treatment using both $\mathrm{CHI}$. Overall survival rates improved from 60 to $85 \%$ compared to monotherapy.

A comparable combination study with pembrolizumab has already been initiated (Table 2). In addition, combination treatment trials with other modulators of inhibitory (e.g., LAG3, HAVCR2, BTLA, and "B and T lymphocyte attenuator") and stimulatory molecules [e.g., ICOS, "inducible T-cell costimulator"; TNFRSF9 (4-1BB)] are under investigation (56).

\section{IMMUNE-CHECKPOINTS AS PROGNOSTIC BIOMARKERS}

Selection markers for targeted therapy with CHI are currently being intensively researched. However, no clear immunological or tumor-specific characteristics could be identified that clearly predict responsiveness to $\mathrm{CHI}$ in $\mathrm{CRC}$ and mCRC. According to current knowledge, the therapy with CHI of both the CTLA4 and the PDCD1/CD247 signaling pathway is particularly well responsive to tumors that have a high mutational load $(57,58)$. Tumors that carry genetic defects in their DNA repair machinery ("MMR defect") and consequently present high mutation rates are much more responsive to anti-PDCD1 therapy than tumors without MMR defects (57). For the PDCD1/CD247 system, most biomarker studies are concerned with the CD247 expression pattern. Looking at all studies across all tumor entities, patients whose tumors express CD247 appear to respond better to
PDCD1 blockade than patients without CD247 expression. Nevertheless, CD247 negative patients also respond to CD247 checkpoint blockade. Therefore, according to current knowledge, CD247 cannot generally be recommended as a selection marker for PDCD1 blockade (56). However, recent data suggest that a patient's CD247 status may play a role in deciding whether to use dual checkpoint inhibition (48). Determining the immunogenicity of the tumor environment could also be important for the choice of tumor therapy. For example, non-immunogenic tumors ("cold tumors") are more likely to benefit from combination therapies (56).

Since CHI activate the adaptive immune system, a tumorspecific immune response is possible, which may be independent of the histological subtype and the type and number of prior therapies. Currently, T-cell therapies alongside $\mathrm{CHI}$ are regarded as great hope carriers of immuno-oncology, even though they are still partially in the developmental phase (45).

\section{T-CELL THERAPIES}

T-cell based immunotherapies are referred to as "live drugs": cell preparations that contain T-cells are currently being clinically researched by oncologists. Adoptive cell transfer (ACT) with Tcells is a highly personalized form of therapy in which patients are endowed with specific T-cells that have direct antitumoral activity. In contrast to vaccination or $\mathrm{CHI}$, the immune system equipped with effector T-cells can exert its antitumoral function immediately. Currently, three classes of effector T-cells are in the process of being approved: tumor-infiltrating lymphocytes (TIL), genetically modified T-cells with a chimeric antigen receptor (CAR), and Tcells targeted with a specific genetically modified $\mathrm{T}$-cell receptor (TCR). In ACT approaches, T-cell lymphocytes are isolated from tumors of individual patients, modified, selected and expanded ex vivo, then reinfused into the patient (Figure 2).

\section{Tumor-Infiltrating Lymphocytes}

It has been shown that the presence of TILs is associated with a good prognosis of cancer and that TILs isolated from tumor tissue show a selective antitumor activity (59).

Preclinical and clinical studies on TIL's ACT show clinical response rates and sustained remission rates in metastatic CRC (60). Recently, modern molecular analysis has shown that melanoma regression-inducing TILs are polyclonal T-cell populations that recognize different neoantigens on tumor cells (61). This finding confirms the long postulated assumption that neoantigens on tumor cells are the main target of immune system recognition (62) and explains the good response rates toward ACT therapies with TILs in melanoma, a tumor entity with one of the highest mutational burdens, or in MSI-H patients (6). Therefore, the establishment of methods for the selection of neoantigen reactive TILs may lead to improved therapeutic success also in other tumor types.

\section{Genetically Modified T-Cells}

The idea of genetically modified T-cells was developed to target directly and more specifically tumor cells with activated T-cells 
that have specific T-cell receptors (TCRs). The production of TCR-modified T-cells is carried out by transfection or transduction of autologous T-cells with vectors, which code for tumor-specific $\alpha / \beta$ TCRs. They can be isolated by different methods $(63,64)$. Any patient whose tumor expresses the tumor antigen and the correct MHC allele may benefit from such therapy approaches. In the complementary strategy of CAR-modified T-cells, CAR genes are expressed in autologous $\mathrm{T}$ lymphocytes. CAR are transmembrane single-chain fusion proteins and the centerpiece of which is an extracellular antibody binding site, which, in contrast to conventional TCR, recognizes an intact surface structure on tumor cells. This Ab binding site is linked to one to three TCR intracytoplasmic signaling regions via a transmembrane region. They serve to initialize T-cell activation signals. CAR modified T-cells are activated and proliferate MHC-independent in vivo after exposure to the antigens. This can lead to tumor cell lysis and the formation of an Ag-specific immune memory (64). Today it is assumed that especially $\mathrm{T}$-cells in early stages of differentiation (naive and central memory T-cells) are particularly suitable for ACT with genemodified T-cells (65). The cancer researchers have succeeded in using genetic engineering to produce third-generation CAR receptors, which can transmit at least three signals, or the fourth generation, known as TRUCK, which can be combined with cytokines $(66,67)$.

To date, CAR therapy has been less successful in solid tumors. In order to be able to better control genetically modified T-cells, it would be advantageous to be able to switch them on and off after infusion. Therefore, Anja Feldmann, Dresden-Rossendorf, and their colleagues have developed special CAR-T-cells that are initially inactive and can be temporarily "armed" only when needed by an externally added factor against tumors. These are short-lived monoor bispecific molecules, for example against the growth factor EGFR ("epidermal growth factor receptor"), which make the connection between the cytotoxic T-cells and the tumor cells and thus the T lymphocytes to the cancer cells. Such UniCAR T-cells can be inactivated again by omitting the activating factors (68).

Several pilot studies with TCR-modified T-cells indicate a good response of various solid and hematological tumors to this therapeutic strategy; these include melanomas, synovial sarcomas, multiple myelomas, colorectal and hepatocellular carcinomas (64). Clinical studies with CAR-modified T-cells have so far been conducted predominantly with anti-ERBB2 specificity for the treatment of CRC/mCRC (69). However, ACT with gene-modified T-cells has often been associated with side effects. Thus, strong immune responses against healthy target tissue could be elicited if the target antigen is not expressed exclusively on tumor cells ("on-target toxicity") or result in cross-reactivities. Neurotoxicities are other frequently observed side effects $(60,64)$. The severe side effects of treatmentinduced massive T-cell activation and associated excessive cytokine secretion, which occur in particular during CAR-modified T-cell therapies, can now be treated well with tocilizumab, a mAb directed against the interleukin-6 receptor (IL6R).

In solid tumors, CAR modified T-cell therapies have not been widely accepted. This is due to the lack of antigens expressed exclusively on tumor cells. In addition, oncological T-cell therapies are extremely costly. Initial clinical trials to combine
ACT with other immuno-oncological therapeutics (CHI and vaccines) or targeted therapies are passing different clinical trials.

\section{IMMUNOTHERAPIES TARGETING CANCER STEM CELLS}

CSCs are resistant to conventional chemotherapies due to their quiescent cell states and are considered the main drivers of disease relapse and cancer metastasis $(70,71)$. In recent studies, a diversity of new antigens has been described that are expressed on colorectal CSCs but are absent in the tumor bulk (non-CSC population) and healthy tissues. These antigens provide promising targets to eradicate CSCs by directed T-cell responses thereby disrupting the generation of new cancer cells. In this regard, the antigen ASB4 has recently been described to be upregulated in colorectal CSCs. Interestingly, treatment with adoptively transferred effector $\mathrm{CD} 8^{+}$ T-cells specifically targeting ASB4 led to the elimination of CSCs and suppressed tumor growth in vivo (71). Glycoprotein A33 (GPA33) was found to be universally expressed on CSCs and non-CSCs populations using a panel of cancer stem-like cell lines derived from human CRC specimens. Treatment with a bispecific GPA33-CD3 monoclonal antibody (MGD007), recruiting human T-cells, induced lysis of GPA33 expressing CRC cells and reduced tumor growth in vivo in NOD/SCID mice subcutaneously injected with a 1:1 mixture of colorectal cancer cell lines (LS174T and Colo205) with purified T-cells (72). The surface markers CD133 and CD44 have been shown to enable the discrimination of colorectal CSCs and non-CSCs $(73,74)$. Radiotheranostic targeting of colorectal CSCs using Prominin 1 (PROM1; known as CD133) and CD44 monoclonal antibodies labeled with radioodine led to a significant inhibition of tumor growth and prolonged mean survival of xenografted mice injected with HT29 CRC cell line (75). Besides immunotherapy-based approaches to target CSCs in CRC/mCRC, novel options are emerging that might play a pivotal role in future treatment regimens targeting and eliminating CSCs. Tankyrase-inhibitors have been shown to effectively reduce the CD44-positive COLO-320DM cell population resembling CSC properties. Moreover, co-treatment of tankyrase-inhibitors with Irinotecan significantly decreased tumor growth of COLO-320 xenograft tumors in immunodeficient mice and showed higher efficiency than single treatment (76). Mithramycin-A (Mit-A) treatment, an antibiotic that inhibits the binding of transcription factors to DNA, led to a reduction in size and numbers of tumor spheroids derived from the CRC cell lines, HT29, HCT116, and KM12 compared to standard treatment with 5-fluorouracil and oxaliplatin (FUOX). In addition, PROM1 expression and ALDH activity of tumor spheroids were downregulated upon Mit-A treatment demonstrating a direct suppressive effect on cancer cell stemness $(77,78)$.

\section{GUT MICROBIOME AND IMMUNOTHERAPIES}

There is emerging evidence that the gut microbiome plays a pivotal role in carcinogenesis, immunity and might affect cancer 
response to immunotherapies $(79,80)$. In CRC, the interactions between gut commensals, immune cells, and cancer cells build a complex and not fully understood network that might drive or inhibit cancer progression depending on various factors such as the composition of the patient's microbiome (Figure 3). In 2009, Wu et al. for the first time described that colonization with entertoxigenic Bacteroides fragilis (BTF) and BTF toxin-mediated colitis followed by recruitment of T helper type 17 (Th17) cells increase the chances of inflammation-induced colorectal cancer (81). The state of the gut microbiome has also been linked to $\mathrm{CHI}$ response in melanoma and renal cell carcinoma patients. In this regard, Chaput et al. described better response rates of patients suffering from metastatic melanoma to treatments with the CTLA4 inhibitor, ipilimumab, based on an intact and stable state of the gut microbiome. Patients whose microbiota was enriched by Faecalibacteria or other Firmicutes survived significantly longer and showed higher rates of ipilimumab-mediated induction of Tcells compared to patients without evidence of gut colonization by Faecalibacteria (82). Moreover, preclinical and clinical studies revealed that treatment with antibiotics disrupting the equilibrium of the normal gut microbiome result in a compromised efficacy of anti-PDCD1 therapies. RET melanoma and MCA-205 sarcoma mice that were pre-treated with antibiotics survived significantly shorter undergoing anti-PDCD1 and antiCTLA4 treatment compared to mice without antibiotic pretreatment. Moreover, in an anti-PDCD1/CD247 treated cohort of 140 non-small cell lung cancer and 67 renal cell carcinoma patients, reduced overall survival could be observed in those that underwent additional antibiotic therapies (83).

Besides the microbiome, many other exogenous and endogenous factors affect cancer progression and therapy response. However, the interplays between these exposures and their effects on cancer progression and therapy response are not well investigated and are therefore an emerging field of scientific interest. The transdisciplinary discipline of molecular pathological epidemiology (MPE) uses molecular pathological signatures to elucidate these complex interactions on disease progression and provide new concepts of disease prediction and treatment. In CRC research, MPE projects led to significant progress in the understanding of cancer heterogeneity between different CRC subtypes based on the analysis and wholesome evaluation of genetic, epigenetic and microbial statuses of CRC patients and have a great potential to improve precision based medicine in the future (84-88).

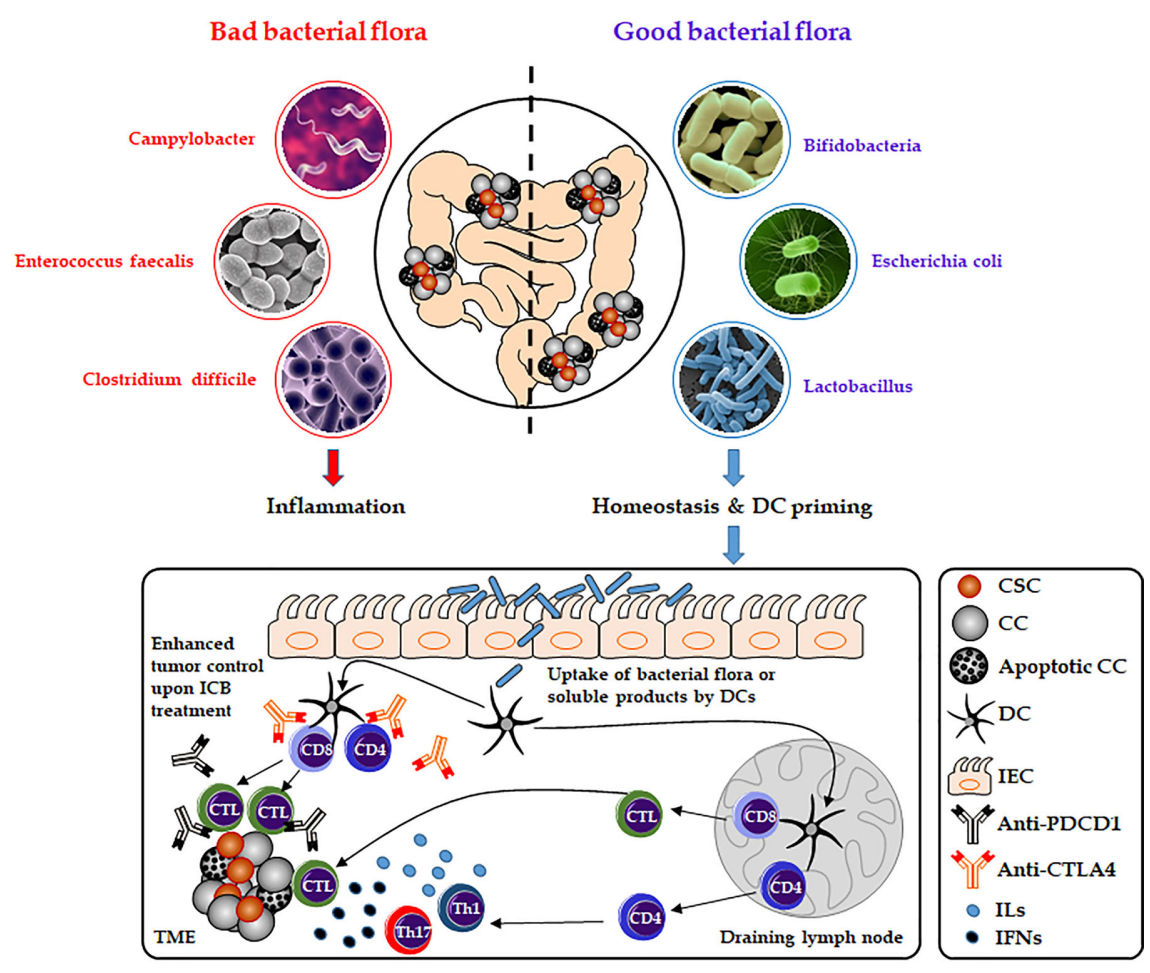

FIGURE 3 | Role of the gut microbiome on immune-checkpoint blockade therapies. Composition of the gut microbiota can affect host antitumor immunity. Uptake of distinct good bacterial flora (e.g., Bifidobacteria, Escherichia coli, and Lactobacillus) or bacteria-derived products by DCs can enhance the antigen-processing steps and presentation by DCs and thereby affect the response to CHI therapies (e.g., anti-PDCD1 or anti-CD247). As a result, this will lead to the activation of cytotoxic T lymphocytes or more increased secretion of interleukins and interferons (e.g., IL17 or INFG) by activated T helper cells. However, bad bacterial species (e.g., Campylobacter, Enterococcus faecalis, and Clostridium difficile) might have a negative effect on DC activation and CHI therapies by inducing inflammation and disrupting the gut microbiome homeostasis. CC, cancer cell; CSC, cancer stem cell; CTL, cytotoxic T lymphocyte; DC, dendritic cell; ICB, immune-checkpoint blockade; IEC, intestinal epithelial cell; IFNs, interferons; ILs, interleukins; Th, T-helper cell; TME, the tumor microenvironment. 


\section{CONCLUSION}

Within a few years, immunotherapy has become a successful oncological therapeutic strategy. It has the potential to induce sustained tumor remission in various tumor entities including $\mathrm{CRC} / \mathrm{mCRC}$, which could significantly improve the overall survival of cancer patients.

Tumor vaccination is highly complex and the optimal combination of antigens, adjuvants and administration routes is not yet clearly identified. From today's perspective, the future of oncological vaccination strategies lies in the development of targeted oncological vaccines based on patient-specific neoantigens and in the combination of various therapeutic strategies such as CHLs, CAR T-cells, or adaptive cell therapies.

Among other developed immunotherapy strategies, checkpoint inhibitors showed a great success rate as a potential immuno-oncological therapy, in particular for dMMR mCRC. However, to date, the economic impact of these therapies largely remains unknown. It is shown that although both single or combination $\mathrm{CHI}$ were superior to chemotherapy in dMMR mCRC, they were less cost-effective.

\section{REFERENCES}

1. Ferlay J, Soerjomataram I, Dikshit R, Eser S, Mathers C, Rebelo M, et al. Cancer incidence and mortality worldwide: sources, methods and major patterns in GLOBOCAN 2012. Int J Cancer (2015) 136(5):E359-86. doi: $10.1002 /$ ijc. 29210

2. Hagness M. Liver transplantation in treatment of colorectal liver metastases. Hepat Oncol (2015) 2(2):181-90. doi: 10.2217/hep.15.3

3. Aghagolzadeh P, Radpour R. New trends in molecular and cellular biomarker discovery for colorectal cancer. World J Gastroenterol (2016) 22(25):5678-93. doi: 10.3748/wjg.v22.i25.5678

4. Feo L, Polcino M, Nash GM. Resection of the Primary Tumor in Stage IV Colorectal Cancer: When Is It Necessary? Surg Clin North Am (2017) 97 (3):657-69. doi: 10.1016/j.suc.2017.01.012

5. van der Geest LG, Lam-Boer J, Koopman M, Verhoef C, Elferink MA, de Wilt JH. Nationwide trends in incidence, treatment and survival of colorectal cancer patients with synchronous metastases. Clin Exp Metastasis (2015) 32 (5):457-65. doi: 10.1007/s10585-015-9719-0

6. Wrobel P, Ahmed S. Current status of immunotherapy in metastatic colorectal cancer. Int J Colorectal Dis (2019) 34(1):13-25. doi: 10.1007/ s00384-018-3202-8

7. Benson AB, Bekaii-Saab T, Chan E, Chen YJ, Choti MA, Cooper HS, et al. Metastatic colon cancer, version 3.2013: featured updates to the NCCN Guidelines. J Natl Compr Canc Netw (2013) 11(2):141-52; quiz 152. doi: 10.6004/jnccn.2013.0022

8. Bredt LC, Rachid AF. Predictors of recurrence after a first hepatectomy for colorectal cancer liver metastases: a retrospective analysis. World J Surg Oncol (2014) 12:391. doi: 10.1186/1477-7819-12-391

9. Pulitano C, Crawford M, Joseph D, Aldrighetti L, Sandroussi C. Preoperative assessment of postoperative liver function: the importance of residual liver volume. J Surg Oncol (2014) 110(4):445-50. doi: 10.1002/jso.23671

10. Sanoff HK, Sargent DJ, Campbell ME, Morton RF, Fuchs CS, Ramanathan RK, et al. Five-year data and prognostic factor analysis of oxaliplatin and irinotecan combinations for advanced colorectal cancer: N9741. J Clin Oncol (2008) 26(35):5721-7. doi: 10.1200/jco.2008.17.7147

11. Visvader JE, Lindeman GJ. Cancer stem cells in solid tumours: accumulating evidence and unresolved questions. Nat Rev Cancer (2008) 8(10):755-68. doi: $10.1038 / \mathrm{nrc} 2499$

12. Riether C, Schurch CM, Flury C, Hinterbrandner M, Druck L, Huguenin AL, et al. Tyrosine kinase inhibitor-induced CD70 expression mediates drug
In $\mathrm{T}$-cell therapies, the response rates vary greatly depending on the underlying disease. Whether and to what extent this benefit can be transferred to other tumors will have to be shown in further studies. In the near future, modern molecular biology techniques might enable the development of patient-specific neoantigen-specific receptors for ACT.

While the role of tumor vaccination is currently unclear, the clinical successes of CHI and ACT with T-cells show that therapeutic manipulation of the immune system represents a new successful oncological treatment strategy for CRC. Cotargeting of CSCs as the disease initiating and maintaining population of cancer cells might increase the success rate of current CRC treatment approaches. Immuno-oncology has the potential to induce sustained tumor regression and significantly improve overall survival in many tumors including mCRC.

\section{AUTHOR CONTRIBUTIONS}

SF and RR wrote the manuscript. All authors contributed to the article and approved the submitted version.

resistance in leukemia stem cells by activating Wnt signaling. Sci Transl Med (2015) 7(298):298ra119. doi: 10.1126/scitranslmed.aab1740

13. Riether C, Schurch CM, Buhrer ED, Hinterbrandner M, Huguenin AL, Hoepner S, et al. CD70/CD27 signaling promotes blast stemness and is a viable therapeutic target in acute myeloid leukemia. J Exp Med (2017) 214 (2):359-80. doi: 10.1084/jem.20152008

14. Radpour R. Tracing and targeting cancer stem cells: new venture for personalized molecular cancer therapy. World J Stem Cells (2017) 9 (10):169-78. doi: 10.4252/wjsc.v9.i10.169

15. Dawood S, Austin L, Cristofanilli M. Cancer stem cells: implications for cancer therapy. Oncol (Williston Park) (2014) 28(12):1101-7, 1110.

16. Murone M, Radpour R, Attinger A, Chessex AV, Huguenin AL, Schurch CM, et al. The Multi-kinase Inhibitor Debio 0617B Reduces Maintenance and Self-renewal of Primary Human AML CD34(+) Stem/Progenitor Cells. Mol Cancer Ther (2017) 16(8):1497-510. doi: 10.1158/15357163.mct-16-0889

17. Radpour R, Forouharkhou F. Single-cell analysis of tumors: Creating new value for molecular biomarker discovery of cancer stem cells and tumor-infiltrating immune cells. World J Stem Cells (2018) 10(11):160-71. doi: 10.4252/ wjsc.v10.i11.160

18. Burnet FM. The concept of immunological surveillance. Prog Exp Tumor Res (1970) 13:1-27. doi: 10.1159/000386035

19. Amedei A, Prisco D, MM DE. The use of cytokines and chemokines in the cancer immunotherapy. Recent Pat Anticancer Drug Discovery (2013) 8 (2):126-42. doi: 10.2174/1574892811308020002

20. Dinarello CA, Ikejima T, Warner SJ, Orencole SF, Lonnemann G, Cannon JG, et al. Interleukin 1 induces interleukin 1. I. Induction of circulating interleukin 1 in rabbits in vivo and in human mononuclear cells in vitro. J Immunol (1987) 139(6):1902-10.

21. Recchia F, Saggio G, Cesta A, Candeloro G, Di Blasio A, Amiconi G, et al. Phase II study of interleukin-2 and 13-cis-retinoic acid as maintenance therapy in metastatic colorectal cancer. Cancer Immunol Immunother (2007) 56(5):699-708. doi: 10.1007/s00262-006-0224-9

22. Rosenberg SA. IL-2: the first effective immunotherapy for human cancer. J Immunol (2014) 192(12):5451-8. doi: 10.4049/jimmunol.1490019

23. Fragale A, Romagnoli G, Licursi V, Buoncervello M, Del Vecchio G, Giuliani C, et al. Antitumor Effects of Epidrug/IFNalpha Combination Driven by Modulated Gene Signatures in Both Colorectal Cancer and Dendritic Cells. Cancer Immunol Res (2017) 5(7):604-16. doi: 10.1158/ 2326-6066.Cir-17-0080 
24. Pfeffer LM, Dinarello CA, Herberman RB, Williams BR, Borden EC, Bordens $\mathrm{R}$, et al. Biological properties of recombinant alpha-interferons: 40th anniversary of the discovery of interferons. Cancer Res (1998) 58(12):2489-99.

25. De Simone V, Franze E, Ronchetti G, Colantoni A, Fantini MC, Di Fusco D, et al. Th17-type cytokines, IL-6 and TNF-alpha synergistically activate STAT3 and NF-kB to promote colorectal cancer cell growth. Oncogene (2015) 34 (27):3493-503. doi: 10.1038/onc.2014.286

26. Heiss MM, Murawa P, Koralewski P, Kutarska E, Kolesnik OO, Ivanchenko $\mathrm{VV}$, et al. The trifunctional antibody catumaxomab for the treatment of malignant ascites due to epithelial cancer: Results of a prospective randomized phase II/III trial. Int J Cancer (2010) 127(9):2209-21. doi: 10.1002/ijc.25423

27. Bargou R, Leo E, Zugmaier G, Klinger M, Goebeler M, Knop S, et al. Tumor regression in cancer patients by very low doses of a $\mathrm{T}$ cell-engaging antibody. Science (2008) 321(5891):974-7. doi: 10.1126/science.1158545

28. Kreiter S, Vormehr M, van de Roemer N, Diken M, Lower M, Diekmann J, et al. Mutant MHC class II epitopes drive therapeutic immune responses to cancer. Nature (2015) 520(7549):692-6. doi: 10.1038/nature14426

29. Makkouk A, Weiner GJ. Cancer immunotherapy and breaking immune tolerance: new approaches to an old challenge. Cancer Res (2015) 75(1):510. doi: 10.1158/0008-5472.Can-14-2538

30. Mellman I, Coukos G, Dranoff G. Cancer immunotherapy comes of age. Nature (2011) 480(7378):480-9. doi: 10.1038/nature10673

31. Frega G, Wu Q, Le Naour J, Vacchelli E, Galluzzi L, Kroemer G, et al. Trial Watch: experimental TLR7/TLR8 agonists for oncological indications. Oncoimmunology (2020) 9(1):1796002. doi: 10.1080/2162402x.2020.1796002

32. Pilch Z, Tonecka K, Braniewska A, Sas Z, Skorzynski M, Boon L, et al. Antitumor Activity of TLR7 Is Potentiated by CD200R Antibody Leading to Changes in the Tumor Microenvironment. Cancer Immunol Res (2018) 6 (8):930-40. doi: 10.1158/2326-6066.Cir-17-0454

33. Kretz-Rommel A, Qin F, Dakappagari N, Ravey EP, McWhirter J, Oltean D, et al. CD200 expression on tumor cells suppresses antitumor immunity: new approaches to cancer immunotherapy. J Immunol (2007) 178(9):5595-605. doi: 10.4049/jimmunol.178.9.5595

34. McWhirter JR, Kretz-Rommel A, Saven A, Maruyama T, Potter KN, Mockridge CII, et al. Antibodies selected from combinatorial libraries block a tumor antigen that plays a key role in immunomodulation. Proc Natl Acad Sci U.S.A. (2006) 103(4):1041-6. doi: 10.1073/pnas.0510081103

35. Krieg AM, Efler SM, Wittpoth M, Al Adhami MJ, Davis HL. Induction of systemic TH1-like innate immunity in normal volunteers following subcutaneous but not intravenous administration of CPG 7909, a synthetic B-class CpG oligodeoxynucleotide TLR9 agonist. J Immunother (2004) 27 (6):460-71. doi: 10.1097/00002371-200411000-00006

36. Klinman DM. Immunotherapeutic uses of $\mathrm{CpG}$ oligodeoxynucleotides. Nat Rev Immunol (2004) 4(4):249-58. doi: 10.1038/nri1329

37. Carpentier AF, Xie J, Mokhtari K, Delattre JY. Successful treatment of intracranial gliomas in rat by oligodeoxynucleotides containing $\mathrm{CpG}$ motifs. Clin Cancer Res (2000) 6(6):2469-73.

38. Nie Y, He J, Shirota H, Trivett AL, Yang D, Klinman DM, et al. Blockade of TNFR2 signaling enhances the immunotherapeutic effect of CpG ODN in a mouse model of colon cancer. Sci Signal (2018) 11(511):eaan0790. doi: 10.1126/ scisignal.aan 0790

39. Vollmer J, Krieg AM. Immunotherapeutic applications of $\mathrm{CpG}$ oligodeoxynucleotide TLR9 agonists. Adv Drug Delivery Rev (2009) 61 (3):195-204. doi: 10.1016/j.addr.2008.12.008

40. Agren J, Thiemermann C, Foster SJ, Wang JE, Aasen AO. Cytokine responses to CpG DNA in human leukocytes. Scand J Immunol (2006) 64(1):61-8. doi: 10.1111/j.1365-3083.2006.01779.x

41. Kissick HT, Sanda MG. The role of active vaccination in cancer immunotherapy: lessons from clinical trials. Curr Opin Immunol (2015) 35:15-22. doi: 10.1016/j.coi.2015.05.004

42. Kobold S, Lutkens T, Cao Y, Bokemeyer C, Atanackovic D. Autoantibodies against tumor-related antigens: incidence and biologic significance. Hum Immunol (2010) 71(7):643-51. doi: 10.1016/j.humimm.2010.03.015

43. Marcucci F, Rumio C, Corti A. Tumor cell-associated immune checkpoint molecules - Drivers of malignancy and stemness. Biochim Biophys Acta Rev Cancer (2017) 1868(2):571-83. doi: 10.1016/j.bbcan.2017.10.006

44. Pardoll DM. The blockade of immune checkpoints in cancer immunotherapy. Nat Rev Cancer (2012) 12(4):252-64. doi: 10.1038/nrc3239
45. Sharma P, Allison JP. The future of immune checkpoint therapy. Science (2015) 348(6230):56-61. doi: 10.1126/science.aaa8172

46. Toor SM, Sasidharan Nair V, Decock J, Elkord E. Immune checkpoints in the tumor microenvironment. Semin Cancer Biol (2020) 65:1-12. doi: 10.1016/ j.semcancer.2019.06.021

47. Hajj C, Russell J, Hart CP, Goodman KA, Lowery MA, Haimovitz-Friedman A, et al. A Combination of Radiation and the Hypoxia-Activated Prodrug Evofosfamide (TH-302) is Efficacious against a Human Orthotopic Pancreatic Tumor Model. Transl Oncol (2017) 10(5):760-5. doi: 10.1016/j.tranon.2017.06.010

48. Le DT, Uram JN, Wang H, Bartlett BR, Kemberling H, Eyring AD, et al. PD-1 Blockade in Tumors with Mismatch-Repair Deficiency. N Engl J Med (2015) 372(26):2509-20. doi: 10.1056/NEJMoa1500596

49. Le DT, Durham JN, Smith KN, Wang H, Bartlett BR, Aulakh LK, et al. Mismatch repair deficiency predicts response of solid tumors to PD-1 blockade. Science (2017) 357(6349):409-13. doi: 10.1126/science.aan6733

50. Overman MJ, McDermott R, Leach JL, Lonardi S, Lenz HJ, Morse MA, et al. Nivolumab in patients with metastatic DNA mismatch repair-deficient or microsatellite instability-high colorectal cancer (CheckMate 142): an openlabel, multicentre, phase 2 study. Lancet Oncol (2017) 18(9):1182-91. doi: 10.1016/s1470-2045(17)30422-9

51. Overman MJ, Lonardi S, Wong KYM, Lenz HJ, Gelsomino F, Aglietta M, et al. Durable Clinical Benefit With Nivolumab Plus Ipilimumab in DNA Mismatch Repair-Deficient/Microsatellite Instability-High Metastatic Colorectal Cancer. J Clin Oncol (2018) 36(8):773-9. doi: 10.1200/jco.2017.76.9901

52. Lee JJ, Chu E. Recent Advances in the Clinical Development of Immune Checkpoint Blockade Therapy for Mismatch Repair Proficient (pMMR)/nonMSI-H Metastatic Colorectal Cancer. Clin Colorectal Cancer (2018) 17 (4):258-73. doi: 10.1016/j.clcc.2018.06.004

53. Kim JH, Kim BS, Lee SK. Regulatory T Cells in Tumor Microenvironment and Approach for Anticancer Immunotherapy. Immune Netw (2020) 20(1):e4. doi: 10.4110/in.2020.20.e4

54. Nishikawa H, Sakaguchi S. Regulatory T cells in cancer immunotherapy. Curr Opin Immunol (2014) 27:1-7. doi: 10.1016/j.coi.2013.12.005

55. Lin YC, Mahalingam J, Chiang JM, Su PJ, Chu YY, Lai HY, et al. Activated but not resting regulatory $\mathrm{T}$ cells accumulated in tumor microenvironment and correlated with tumor progression in patients with colorectal cancer. Int $J$ Cancer (2013) 132(6):1341-50. doi: 10.1002/ijc.27784

56. Hodi FS, O'Day SJ, McDermott DF, Weber RW, Sosman JA, Haanen JB, et al. Improved survival with ipilimumab in patients with metastatic melanoma. $N$ Engl J Med (2010) 363(8):711-23. doi: 10.1056/NEJMoa1003466

57. Schumacher TN, Schreiber RD. Neoantigens in cancer immunotherapy. Science (2015) 348(6230):69-74. doi: 10.1126/science.aaa4971

58. Rizvi NA, Hellmann MD, Snyder A, Kvistborg P, Makarov V, Havel JJ, et al. Cancer immunology. Mutational landscape determines sensitivity to PD-1 blockade in non-small cell lung cancer. Science (2015) 348(6230):124-8. doi: $10.1126 /$ science.aaal 348

59. Rosenberg SA, Restifo NP. Adoptive cell transfer as personalized immunotherapy for human cancer. Science (2015) 348(6230):62-8. doi: 10.1126/science.aaa4967

60. Fan J, Shang D, Han B, Song J, Chen H, Yang JM. Adoptive Cell Transfer: Is it a Promising Immunotherapy for Colorectal Cancer? Theranostics (2018) 8 (20):5784-800. doi: 10.7150/thno.29035

61. Dudley ME, Yang JC, Sherry R, Hughes MS, Royal R, Kammula U, et al. Adoptive cell therapy for patients with metastatic melanoma: evaluation of intensive myeloablative chemoradiation preparative regimens. J Clin Oncol (2008) 26(32):5233-9. doi: 10.1200/jco.2008.16.5449

62. Lennerz V, Fatho M, Gentilini C, Frye RA, Lifke A, Ferel D, et al. The response of autologous $\mathrm{T}$ cells to a human melanoma is dominated by mutated neoantigens. Proc Natl Acad Sci U.S.A. (2005) 102(44):16013-8. doi: 10.1073/pnas.0500090102

63. Morgan RA, Dudley ME, Wunderlich JR, Hughes MS, Yang JC, Sherry RM, et al. Cancer regression in patients after transfer of genetically engineered lymphocytes. Science (2006) 314(5796):126-9. doi: 10.1126/science.1129003

64. Barrett DM, Grupp SA, June CH. Chimeric Antigen Receptor- and TCRModified T Cells Enter Main Street and Wall Street. J Immunol (2015) 195 (3):755-61. doi: 10.4049/jimmunol.1500751

65. June $\mathrm{CH}$, Riddell SR, Schumacher TN. Adoptive cellular therapy: a race to the finish line. Sci Transl Med (2015) 7(280):280ps7. doi: 10.1126/scitranslmed. aaa3643 
66. Chmielewski M, Abken H. TRUCKs: the fourth generation of CARs. Expert Opin Biol Ther (2015) 15(8):1145-54. doi: 10.1517/14712598.2015.1046430

67. Martinez M, Moon EK. CAR T Cells for Solid Tumors: New Strategies for Finding, Infiltrating, and Surviving in the Tumor Microenvironment. Front Immunol (2019) 10:128. doi: 10.3389/fimmu.2019.00128

68. Feldmann A, Arndt C, Koristka S, Berndt N, Bergmann R, Bachmann MP. Conventional CARs versus modular CARs. Cancer Immunol Immunother (2019) 68(10):1713-9. doi: 10.1007/s00262-019-02399-5

69. Duong CP, Yong CS, Kershaw MH, Slaney CY, Darcy PK. Cancer immunotherapy utilizing gene-modified T cells: From the bench to the clinic. Mol Immunol (2015) 67(2 Pt A):46-57. doi: 10.1016/.jmolimm.2014.12.009

70. Li X, Lewis MT, Huang J, Gutierrez C, Osborne CK, Wu MF, et al. Intrinsic resistance of tumorigenic breast cancer cells to chemotherapy. J Natl Cancer Inst (2008) 100(9):672-9. doi: 10.1093/jnci/djn123

71. Miyamoto S, Kochin V, Kanaseki T, Hongo A, Tokita S, Kikuchi Y, et al. The Antigen ASB4 on Cancer Stem Cells Serves as a Target for CTL Immunotherapy of Colorectal Cancer. Cancer Immunol Res (2018) 6 (3):358-69. doi: 10.1158/2326-6066.Cir-17-0518

72. Moore PA, Shah K, Yang Y, Alderson R, Roberts P, Long V, et al. Development of MGD007, a gpA33 x CD3-Bispecific DART Protein for TCell Immunotherapy of Metastatic Colorectal Cancer. Mol Cancer Ther (2018) 17(8):1761-72. doi: 10.1158/1535-7163.Mct-17-1086

73. Haraguchi N, Ohkuma M, Sakashita H, Matsuzaki S, Tanaka F, Mimori K, et al. CD133+CD44+ population efficiently enriches colon cancer initiating cells. Ann Surg Oncol (2008) 15(10):2927-33. doi: 10.1245/s10434-008-0074-0

74. Sahlberg SH, Spiegelberg D, Glimelius B, Stenerlow B, Nestor M. Evaluation of cancer stem cell markers CD133, CD44, CD24: association with AKT isoforms and radiation resistance in colon cancer cells. PloS One (2014) 9(4):e94621. doi: 10.1371/journal.pone.0094621

75. She X, Qin S, Jing B, Jin X, Sun X, Lan X, et al. Radiotheranostic Targeting Cancer Stem Cells in Human Colorectal Cancer Xenografts. Mol Imaging Biol (2020) 22(4):1043-53. doi: 10.1007/s11307-019-01467-7

76. Jang MK, Mashima T, Seimiya H. Tankyrase Inhibitors Target Colorectal Cancer Stem Cells via AXIN-Dependent Downregulation of c-KIT Tyrosine Kinase. Mol Cancer Ther (2020) 19(3):765-76. doi: 10.1158/1535-7163.Mct19-0668

77. Barcelo F, Ortiz-Lombardia M, Martorell M, Oliver M, Mendez C, Salas JA, et al. DNA binding characteristics of mithramycin and chromomycin analogues obtained by combinatorial biosynthesis. Biochemistry (2010) 49 (49):10543-52. doi: 10.1021/bi101398s

78. Quarni W, Dutta R, Green R, Katiri S, Patel B, Mohapatra SS, et al. Mithramycin A Inhibits Colorectal Cancer Growth by Targeting Cancer Stem Cells. Sci Rep (2019) 9(1):15202. doi: 10.1038/s41598-019-50917-3
79. Gopalakrishnan V, Helmink BA, Spencer CN, Reuben A, Wargo JA. The Influence of the Gut Microbiome on Cancer, Immunity, and Cancer Immunotherapy. Cancer Cell (2018) 33(4):570-80. doi: 10.1016/j.ccell.2018.03.015

80. Sears CL, Garrett WS. Microbes, microbiota, and colon cancer. Cell Host Microbe (2014) 15(3):317-28. doi: 10.1016/j.chom.2014.02.007

81. Wu S, Rhee KJ, Albesiano E, Rabizadeh S, Wu X, Yen HR, et al. A human colonic commensal promotes colon tumorigenesis via activation of $\mathrm{T}$ helper type 17 T cell responses. Nat Med (2009) 15(9):1016-22. doi: 10.1038/nm.2015

82. Chaput N, Lepage P, Coutzac C, Soularue E, Le Roux K, Monot C, et al. Baseline gut microbiota predicts clinical response and colitis in metastatic melanoma patients treated with ipilimumab. Ann Oncol (2017) 28(6):136879. doi: $10.1093 /$ annonc/mdx108

83. Routy B, Le Chatelier E, Derosa L, Duong CPM, Alou MT, Daillère R, et al. Gut microbiome influences efficacy of PD-1-based immunotherapy against epithelial tumors. Science (2018) 359(6371):91-7. doi: 10.1126/science.aan3706

84. Ogino S, Chan AT, Fuchs CS, Giovannucci E. Molecular pathological epidemiology of colorectal neoplasia: an emerging transdisciplinary and interdisciplinary field. Gut (2011) 60(3):397-411. doi: 10.1136/gut.2010.217182

85. Punt CJ, Koopman M, Vermeulen L. From tumour heterogeneity to advances in precision treatment of colorectal cancer. Nat Rev Clin Oncol (2017) 14 (4):235-46. doi: 10.1038/nrclinonc.2016.171

86. Riley JM, Cross AW, Paulos CM, Rubinstein MP, Wrangle J, Camp ER. The clinical implications of immunogenomics in colorectal cancer: A path for precision medicine. Cancer (2018) 124(8):1650-9. doi: 10.1002/cncr.31214

87. Dienstmann R, Vermeulen L, Guinney J, Kopetz S, Tejpar S, Tabernero J. Consensus molecular subtypes and the evolution of precision medicine in colorectal cancer. Nat Rev Cancer (2017) 17(2):79-92. doi: 10.1038/ nrc.2016.126

88. Hamada T, Nowak JA, Milner DAJr., Song M, Ogino S. Integration of microbiology, molecular pathology, and epidemiology: a new paradigm to explore the pathogenesis of microbiome-driven neoplasms. J Pathol (2019) 247(5):615-28. doi: 10.1002/path.5236

Conflict of Interest: The authors declare that the research was conducted in the absence of any commercial or financial relationships that could be construed as a potential conflict of interest.

Copyright $\odot 2020$ Forster and Radpour. This is an open-access article distributed under the terms of the Creative Commons Attribution License (CC BY). The use, distribution or reproduction in other forums is permitted, provided the original author(s) and the copyright owner(s) are credited and that the original publication in this journal is cited, in accordance with accepted academic practice. No use, distribution or reproduction is permitted which does not comply with these terms. 